\title{
Post-exercise impact of ingested whey protein hydrolysate on gene expression profiles in rat skeletal muscle: activation of extracellular signal-regulated kinase $1 / 2$ and hypoxia-inducible factor- $1 \alpha$
}

\author{
Atsushi Kanda ${ }^{1 *}$, Tomoko Ishijima ${ }^{2,3}$, Fumika Shinozaki ${ }^{3}$, Kyosuke Nakayama $^{1}$, Tomoyuki Fukasawa ${ }^{1}$, \\ Yuji Nakai ${ }^{2,3}$, Keiko Abe ${ }^{2,3}$, Keiko Kawahata ${ }^{1}$ and Shuji Ikegami ${ }^{1}$ \\ ${ }^{1}$ Food Science Research Laboratories, Meiji Company Limited, 540 Naruda, Odawara, Kanagawa 250-0862, Japan \\ ${ }^{2}$ Department of Applied Biological Chemistry, Graduate School of Agricultural and Life Sciences, \\ The University of Tokyo, 1-1-1 Yayoi, Bunkyo-ku, Tokyo 113-8657, Japan \\ ${ }^{3}$ Project on Health and Anti-aging, Kanagawa Academy of Science and Technology, Life Science and Environment \\ Research Center 4F C-4, 3-25-13 Tonomachi, Kawasaki-ku, Kawasaki, Kanagawa 210-0821, Japan \\ (Submitted 18 September 2013 - Final revision received 10 December 2013 - Accepted 9 January 2014 - First published online 6 March 2014)
}

\begin{abstract}
We have previously shown that whey protein hydrolysate (WPH) causes a greater increase in muscle protein synthesis than does a mixture of amino acids that is identical in amino acid composition. The present study was conducted to investigate the effect of WPH on gene expression. Male Sprague-Dawley rats subjected to a $2 \mathrm{~h}$ swimming exercise were administered either a carbohydrate-amino acid diet or a carbohydrate-WPH diet immediately after exercise. At $1 \mathrm{~h}$ after exercise, epitrochlearis muscle mRNA was sampled and subjected to DNA microarray analysis. We found that ingestion of WPH altered 189 genes after considering the false discovery rate. Among the up-regulated genes, eight Gene Ontology (GO) terms were enriched, which included key elements such as $\mathrm{Cd} 24$, Ccl2, Ccl7 and $\mathrm{Cxcl1}$ involved in muscle repair after exercise. In contrast, nine GO terms were enriched in gene sets that were down-regulated by the ingestion of WPH, and these GO terms fell into two clusters, 'regulation of ATPase activity' and 'immune response'. Furthermore, we found that WPH activated two upstream proteins, extracellular signal-regulated kinase $1 / 2$ (ERK1/2) and hypoxia-inducible factor- $1 \alpha$ (HIF-1 $\alpha)$, which might act as key factors for regulating gene expression. These results suggest that ingestion of WPH, compared with ingestion of a mixture of amino acids with an identical amino acid composition, induces greater changes in the post-exercise gene expression profile via activation of the proteins ERK1/2 and HIF-1 $\alpha$.
\end{abstract}

Key words: Whey protein hydrolysates: DNA microarray analysis: Exercise: Amino acids

Prolonged exercise induces physiological changes, especially in terms of muscle glycogen depletion ${ }^{(1)}$ and the regulation of skeletal muscle protein synthesis ${ }^{(2,3)}$. Studies of recovery after strenuous exercise have focused on the repletion of tissue carbohydrate and protein ${ }^{(2,4)}$.

Dietary carbohydrate is an effective source of tissue glycogen. Several studies ${ }^{(4,5)}$ have reported that consuming a combination of carbohydrates and proteins after exercise replenishes muscle glycogen more effectively than does consuming carbohydrates alone. Recent studies ${ }^{(6-8)}$ have demonstrated that amino acids, in particular branched-chain amino acids (BCAA), especially leucine, stimulate glycogen synthesis in skeletal muscle.
During exercise, muscle protein synthesis is inhibited. However, within the first hour following exercise, muscle protein synthesis is enhanced and the increase can persist for 24 to $48 \mathrm{~h}^{(2,3)}$. In addition, previous studies have demonstrated that ingestion of a meal containing protein immediately following exercise stimulates protein synthesis in skeletal muscle $\mathrm{e}^{(2)}$ In particular, leucine alone stimulates muscle protein synthesis to the same extent as complete protein or a complete mixture of amino acids ${ }^{(9,10)}$. This effect is due to a dose-dependent activation of several factors involved in the initiation of mRNA translation, and is mediated primarily through the mammalian target of rapamycin (mTOR) signalling pathway ${ }^{(11,12)}$

Abbreviations: AAM, amino acid mixture; BCAA, branched-chain amino acids; $C c l 7$, chemokine (C-C motif) ligand 7; DEG, differentially expressed genes; DFW, distribution free weighted; Egr1, early growth response 1; ERK1/2, extracellular signal-regulated kinase 1/2; FSR, fractional rate of protein synthesis; GO, Gene Ontology; Hmox1, haem oxygenase (decycling) 1; HIF-1 $\alpha$, hypoxia-inducible factor-1 $\alpha$; IPA, Ingenuity Pathways Analysis; Junb, Jun B protooncogene; mTOR, mammalian target of rapamycin; $N r 4 a 1$, nuclear receptor subfamily 4 , group A, member $1 ; N r 4 a 3$, nuclear receptor subfamily 4 , group A, member 3; WPH, whey protein hydrolysate.

* Corresponding author: A. Kanda, fax +81 46537 3638, email atsushi.kanda.ba@meiji.com 
These studies have indicated that the effect of dietary protein on muscle protein synthesis is due to its leucine content ${ }^{(13)}$.

We have shown that not only the BCAA content but also the molecular forms of dietary protein are an effective factor after exercise ${ }^{(14,15)}$. In this regard, enzymatically hydrolysed whey protein (whey protein hydrolysate (WPH)) is of particular interest. We have previously demonstrated that BCAA-containing peptides in WPH increase the rate of glucose uptake in vitro ${ }^{(16)}$. Ingestion of carbohydrate plus WPH increases glycogen content in the rat skeletal muscle in a $2 \mathrm{~h}$ period after exercise more than intact whey protein or $\mathrm{BCAA}^{(14)}$. Furthermore, we have shown that ingestion of WPH causes a greater increase in muscle protein synthesis and phosphorylation levels of $\mathrm{mTOR}^{(15)}$. These results indicate that WPH is superior to amino acids in stimulating muscle glycogen synthesis and muscle protein synthesis.

Recently, Rowlands et al. ${ }^{(17)}$ showed that post-exercise ingestion of whey protein differentially alters the portion of the transcriptome that is involved in tissue structure and remodelling, including production of the extracellular matrix, cytoskeleton and contractile proteins. However, there is only limited information on the differential effects of protein hydrolysates $v$. amino acids on post-exercise gene expression in skeletal muscle. Thus, the possibility exists that WPH has novel effects on gene expression. To test this hypothesis, we investigated the global effect of WPH on gene expression in skeletal muscle in comparison with an identical amino acid mixture (AAM).

\section{Materials and methods}

\section{Animals}

The animals used in the present study were the same as those used in our previous study ${ }^{(15)}$. Briefly, male Sprague-Dawley rats ( 5 weeks old) with a body weight of approximately $150 \mathrm{~g}$ (CLEA Japan, Inc.) were used. Rats were maintained at $23 \pm 2^{\circ} \mathrm{C}$, with lights on from 08.00 to 20.00 hours and off from 20.00 to 08.00 hours. Rats had free access to food (protein $23.6 \%$, fat $5.3 \%$, carbohydrate $54.4 \%$, ash $6.1 \%$, fibre $2.9 \%$, moisture $7.7 \%$, MF; Oriental Yeast Company Limited) and water. The present study was approved by the Animal Committee of Food Science Research Laboratory, Meiji Company Limited, and followed their guidelines for the care and use of animals.

\section{Experimental protocol}

A previously described experimental protocol was used ${ }^{(15)}$. Briefly, after 2 or $3 \mathrm{~d}$ of acclimatisation, rats were pre-trained to undergo swimming exercise for $3 \mathrm{~d}$. On $1 \mathrm{~d}$ before the experiment, they were fed $5 \mathrm{~g}$ of a restricted diet (MF; Oriental Yeast Company Limited) to control food intake according to previous studies $^{(14,18)}$. On the day of the experiment, rats swam for $2 \mathrm{~h}$, with four rats swimming simultaneously in a barrel filled with water to a depth of $50 \mathrm{~cm}$, allowing an average surface area of $400 \mathrm{~cm}^{2}$ for each animal. Rats started to swim between 10.00 and 12.00 hours. The water temperature was maintained at a constant of $35^{\circ} \mathrm{C}$. Immediately following exercise, rats were given one of two isoenergetic test solutions by oral administration ( $n 8$ per group). These solutions contained $44 \mathrm{~kJ}$ in a four-test dose that represented about $15 \%$ of daily energy needs as determined previously, and included either a mixed meal containing carbohydrate and an AAM or a mixed meal containing carbohydrate and WPH (Meiji Company Limited). WPH used in the present study was a WPH generated by a protease from Bacillus sp. The WPH contained $21.6 \mathrm{mg} / \mathrm{g}$ of the following BCAA-containing bioactive peptides: Ile-Val; Leu-Val; Val-Leu; Ile-Ile; Ile-Leu; Leu-Ile; Leu-Leu ${ }^{(16)}$. Each test solution was isoenergetic $(1100 \mathrm{~kJ} / 100 \mathrm{ml})$, and contained equal amounts of protein, with the protein content of these preparations being measured by the Kjeldahl method ${ }^{(15)}$. The average chain length of the peptides was calculated as the ratio of total $\mathrm{N}$ :amino $\mathrm{N}$ in the protein samples, which showed that WPH had an average peptide length of 3.64 residues. The composition of the AAM was equivalent to that of WPH, which was prepared by mixing pure crystalline L-amino acids and glycine (Wako Pure Chemical Industries) ${ }^{(15)}$.

Exactly $1 \mathrm{~h}$ after administration of the test solutions, rats were killed under diethyl ether anaesthesia, and the epitrochlearis and triceps muscles were excised. The epitrochlearis muscle was stored in RNAlater (Qiagen K.K.) at $4^{\circ} \mathrm{C}$ for $15 \mathrm{~h}$ and then at $-20^{\circ} \mathrm{C}$ until use. The triceps muscle was stored at $-80^{\circ} \mathrm{C}$ until further use.

\section{Measurement of the fractional rates of protein synthesis}

The experimental protocol was the same as described previously $^{(15)}$. Briefly, $15 \mathrm{~min}$ before killing, a bolus dose ( $45 \mathrm{mg} / \mathrm{kg}$ body weight, $22.5 \mathrm{mg} / \mathrm{ml}$ ) of ${ }^{2} \mathrm{H}$-labelled phenylalanine ( $\left[{ }^{2} \mathrm{H}_{5}\right]$ Phe; Cambridge Isotope Laboratories) was injected via the tail vein for the measurement of fractional rate of protein synthesis (FSR). At $15 \mathrm{~min}$ after the injection, the triceps muscle was excised and frozen rapidly. $\left[{ }^{2} \mathrm{H}_{5}\right] \mathrm{Phe}$ enrichment in plasma and hydrolysed muscle protein samples was determined using an LC/MS/MS system (TQD; Waters Corporation). FSR, defined as the percentage of tissue protein renewed each day, was calculated according to the formula:

$$
\mathrm{FSR}=\left(E_{\mathrm{b}} \times 100\right) /\left(E_{\mathrm{a}} \times t\right),
$$

where $t$ is the time interval between the injection and cooling of the tissue sample, expressed in $\mathrm{d}$, and $E_{\mathrm{b}}$ and $E_{\mathrm{a}}$ are the enrichments of $\left[{ }^{2} \mathrm{H}_{5}\right]$ Phe in the hydrolysed tissue protein and in muscle free amino acids, respectively.

\section{Measurement of plasma insulin and amino acids}

The experimental protocol was the same as described previously ${ }^{(15)}$. Briefly, plasma insulin was measured using a commercial ELISA kit (Mercodia AB). Plasma free amino acids were assessed by HPLC, with pre-column derivatisation by 6-aminoquinolyl- $N$-hydroxysuccinimidyl carbamate ${ }^{(19)}$.

\section{DNA microarray experiments}

From each experimental group, four rats were selected according to the average FSR and subjected to DNA 
microarray analysis (Table 1). RNA was extracted from each epitrochlearis muscle using the Qiagen RNeasy Fibrous Tissue Mini Kit (Qiagen). The appropriateness of the quality and quantity of RNA samples was confirmed by the measurement of optical densities at 260 and $280 \mathrm{~nm}$ as well as by the observation of gel electrophoresis patterns obtained using a BioAnalyzer (Agilent Technologies). DNA microarray analysis was carried out as described previously ${ }^{(20)}$. In brief, biotinylated antisense RNA was obtained from 100 ng of purified total RNA using a GeneChip $3^{\prime}$ IVT Express Kit (Affymetrix). The antisense RNA was purified, fragmented and then hybridised to an Affymetrix Rat Genome 230.2.0 Array containing probe sets for over 30000 rat genes. Following hybridisation at $45^{\circ} \mathrm{C}$ for $16 \mathrm{~h}$, the arrays were washed and labelled with phycoerythrin. Fluorescence signals were scanned using the Affymetrix GeneChip System. Affymetrix GeneChip Command Console software was used to convert the array images to the intensity of each probe (CEL files). All the microarray data are MIAME compliant and have been deposited in a MIAME compliant database, the National Center for Biotechnology Information (NCBI) Gene Expression Omnibus (http://www.ncbi.nlm.nih. gov/geo/, GEO Series accession no. GSE49486), as detailed on the MGED Society website (http://www.mged.org/Work groups/MIAME/miame.html).

\section{DNA microarray data analysis}

CEL files were quantified with the distribution free weighted (DFW) method ${ }^{(21)}$ using statistical language R (http://www. r-project) ${ }^{(22)}$ and Bioconductor (http://www.bioconductor. org/ $)^{(23)}$. Principal component analysis ${ }^{(24)}$ was performed using the prcomp() function in R. To identify differentially expressed genes (DEG), the rank products method ${ }^{(25)}$ was applied to DFW-quantified data, with the number of permutations set at 500. Probe sets with a false discovery rate $<0.05$ were regarded as having different expression levels

Table 1. Fractional rate of protein synthesis (FSR) and plasma parameters

(Mean values with their standard errors)

\begin{tabular}{|c|c|c|c|c|}
\hline & \multicolumn{2}{|c|}{ AAM } & \multicolumn{2}{|c|}{ WPH } \\
\hline & Mean & SE & Mean & $\mathrm{SE}$ \\
\hline \multicolumn{5}{|c|}{ Rats ingested an AAM or WPH after exercise $(n 8) \dagger$} \\
\hline FSR $(\% / d)$ & $5 \cdot 8$ & $0 \cdot 3$ & $6 \cdot 9^{\star}$ & $0 \cdot 1$ \\
\hline Body weight (g) & $169 \cdot 6$ & $2 \cdot 0$ & $169 \cdot 9$ & $2 \cdot 1$ \\
\hline Plasma insulin $(\mathrm{pmol} / \mathrm{l})$ & $107 \cdot 9$ & $20 \cdot 1$ & 147.9 & $20 \cdot 4$ \\
\hline Plasma TAA $(\mathrm{mmol} / \mathrm{l})$ & 7.4 & 0.5 & $7 \cdot 1$ & 0.2 \\
\hline Plasma BCAA (mmol/l) & $1 \cdot 1$ & $0 \cdot 1$ & $1 \cdot 1$ & $0 \cdot 1$ \\
\hline \multicolumn{5}{|c|}{ Rats subjected to DNA microarray analysis $(n 4)$} \\
\hline FSR $(\% / d)$ & $5 \cdot 8$ & 0.2 & $6 \cdot 9^{*}$ & $0 \cdot 1$ \\
\hline Body weight $(\mathrm{g})$ & $166 \cdot 7$ & $1 \cdot 2$ & $172 \cdot 0$ & $2 \cdot 7$ \\
\hline Plasma insulin (pmol/l) & 83.3 & $13 \cdot 6$ & $126 \cdot 9$ & 11.3 \\
\hline Plasma TAA $(\mathrm{mmol} / \mathrm{l})$ & $6 \cdot 9$ & 0.4 & $7 \cdot 2$ & 0.3 \\
\hline Plasma BCAA (mmol/l) & $1 \cdot 1$ & $0 \cdot 1$ & $1 \cdot 2$ & $0 \cdot 1$ \\
\hline
\end{tabular}

AAM, amino acid mixture; WPH, whey protein hydrolysate; TAA, total amino acids; BCAA, branched-chain amino acids.

* Mean value was significantly different from that of the AAM group $(P<0.05$; Student's $t$ test).

$\dagger$ These data are cited from our previous study ${ }^{(15)}$. between the two groups (i.e. they were differently expressed). The annotation file for the Rat Genome 230.2.0 Array was downloaded from the Affymetrix web site (29 October 2012, Rat230_2.na33.annot.csv).

Gene-annotation enrichment analysis of DEG was performed using the Database for Annotation, Visualisation and Integrated Discovery (DAVID; http://david.abcc.ncifcrf.gov/) ${ }^{(26)}$ and Quick Gene Ontology (GO) (http://www.ebi.ac.uk/QuickGO/) ${ }^{(27)}$.

EASE (Expression Analysis Systematic Explorer) scores, which are modified Fisher's exact test $P$ values ${ }^{(28)}$, were used to extract statistically over-represented GO terms from DEG. GO terms with $P$ values $<0.01$ were regarded as significantly enriched.

\section{Analysis of gene networks}

Gene networks from DEG were generated using Ingenuity Pathways Analysis (IPA; Ingenuity Systems, http://www.ingenuity. com). A gene network can be represented as a graph in which gene products are represented as nodes, and the biological relationship between two nodes is represented as an edge (line). All edges are supported by at least one reference from the literature, from a textbook or from canonical information stored in the Ingenuity Pathways Knowledge Base. The intensity of the node colour indicates the degree of up-regulation (red) or down-regulation (green). Nodes are displayed using various shapes that represent the functional class of the gene product. DEG have been overlaid onto these constructed networks to further understand specific interactions among genes of interest.

\section{Prediction of upstream regulators and transcription factors from differentially expressed genes}

Upstream regulators and transcription factors of DEG were predicted using IPA and TFactS (http://www.tfacts.org) ${ }^{(29,30)}$, respectively. IPA uses a right-tailed Fisher's exact test to calculate a $P$ value, determining the probability that each upstream regulator assigned to the dataset occurred by chance. TFactS compares submitted gene lists with annotated target genes in the catalogue to detect regulated transcription factors.

\section{Western blotting}

Triceps muscle supernatants were subjected to Western blotting as described previously ${ }^{(31)}$. Phosphorylation of extracellular signal-regulated kinase 1/2 (ERK1/2) at Thr202/Tyr204 was detected using rabbit anti-phospho-ERK1/2 (Thr202/Tyr204) (Cell Signaling Technology), and is expressed as a ratio to total ERK1/2 expression, determined using an anti-ERK1/2 antibody (Cell Signaling Technology). Hypoxia-inducible factor-1 $\alpha$ (HIF-1 $\alpha)$ was detected using a rabbit anti-HIF- $1 \alpha$ antibody (Santa Cruz Biotechnology), and is expressed as the ratio of $\beta$-actin expression, determined using an anti- $\beta$-actin antibody (Cell Signaling Technology).

\section{Statistical analysis}

FSR and plasma parameters are expressed as means with their standard errors. Comparison of the data was carried 
(a)

\begin{tabular}{|c|c|c|}
\hline GO ID & GO term & $P$ \\
\hline 0006955 & :..... Immune response & $7 \cdot 80 \times 10^{-5}$ \\
\hline 0001818 & ....................... & $2.33 \times 10^{-3}$ \\
\hline 0051252 & .......................... Regulation of RNA metabolic process & $4.81 \times 10^{-3}$ \\
\hline 0051254 & i...... Positive regulation of RNA metabolic process & $2 \cdot 24 \times 10^{-3}$ \\
\hline 0045893 & :..... Positive regulation of transcription, DNA-dependent & $2.08 \times 10^{-3}$ \\
\hline 0045944 & $\begin{array}{l}\text { positive regulation of transcription from RNA } \\
\text { polymerase II promoter }\end{array}$ & $6.05 \times 10^{-3}$ \\
\hline 0006357 & :- Regulation of transcription from RNA polymerase II promoter & $7 \cdot 72 \times 10^{-4}$ \\
\hline 0006355 & Regulation of transcription, DNA-dependent & $3.57 \times 10^{-3}$ \\
\hline 0010628 & Positive regulation of gene expression & $8.73 \times 10^{-3}$ \\
\hline 0051960 & .................. Regulation of nervous system development & $5.00 \times 10^{-3}$ \\
\hline 0045664 & 3........ Regulation of neuron differentiation & $4 \cdot 19 \times 10^{-3}$ \\
\hline 0045665 & :... Negative regulation of neuron differentiation & $3.48 \times 10^{-3}$ \\
\hline 0010033 & .......... Response to organic substance & $5 \cdot 35 \times 10^{-3}$ \\
\hline 0009612 & ......... Response to mechanical stimulus & $5 \cdot 31 \times 10^{-3}$ \\
\hline 0009611 & .......... Response to wounding & $5.97 \times 10^{-3}$ \\
\hline 0060537 & a.............. Muscle tissue development & $8.47 \times 10^{-3}$ \\
\hline 0014706 & a... Striated muscle tissue development & $6 \cdot 70 \times 10^{-3}$ \\
\hline
\end{tabular}

(b)

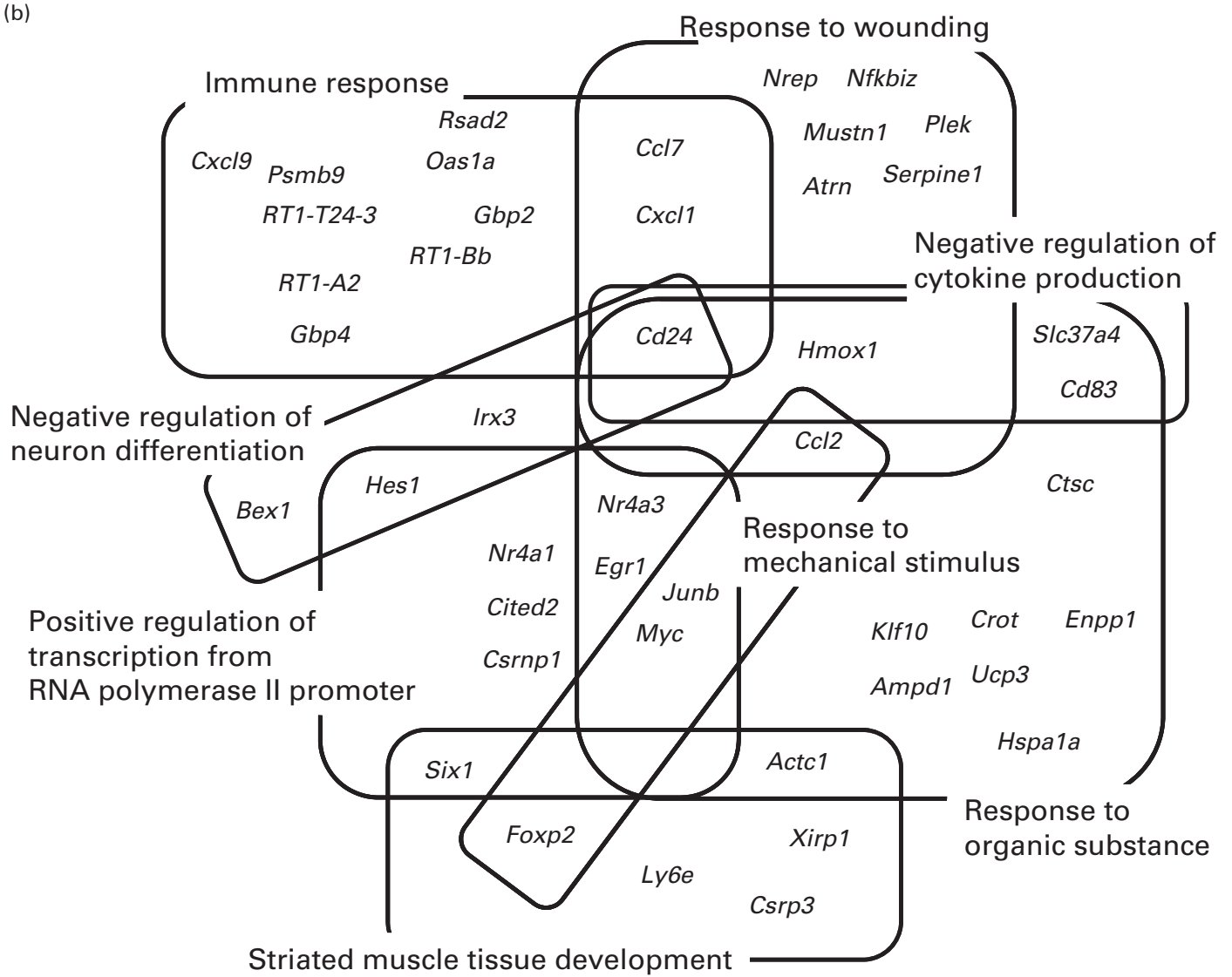

Fig. 1. Significantly enriched Gene Ontology $(G O)$ terms $(P<0.01)$ found in the top 161 up-regulated genes in the whey protein hydrolysate (WPH) group (a). Venn and Euler diagrams represent the association of up-regulated genes with multiple GO terms caused by WPH (b). For full names of gene symbols, see online supplementary Table $\mathrm{S} 1$. 
(a)

\begin{tabular}{|c|c|c|}
\hline GO ID & GO term & $P$ \\
\hline 0006955 & :..... Immune response & $3 \cdot 24 \times 10^{-3}$ \\
\hline 0019882 & ...... Antigen processing and presentation & $1 \cdot 24 \times 10^{-3}$ \\
\hline 0048002 & A... Antigen processing and presentation of peptide antigen & $3 \cdot 28 \times 10^{-4}$ \\
\hline 0002474 & 3... Antigen processing and presentation of peptide antigen via $\mathrm{MHC}$ class I & $2 \cdot 34 \times 10^{-3}$ \\
\hline 0003012 & ................ Muscle system process & $8.79 \times 10^{-4}$ \\
\hline 0006936 & !... Muscle contraction & $5.52 \times 10^{-4}$ \\
\hline 0006941 & ¿... Striated muscle contraction & $2.46 \times 10^{-5}$ \\
\hline 0003013 & a..... Circulatory system process & $4.45 \times 10^{-3}$ \\
\hline 0008015 & !... Blood circulation & $4.45 \times 10^{-3}$ \\
\hline 0060537 & .... Muscle tissue development & $2.59 \times 10^{-4}$ \\
\hline 0014706 & W....... Striated muscle tissue development & $1.99 \times 10^{-4}$ \\
\hline 0048738 & ......... Cardiac muscle tissue development & $1.86 \times 10^{-3}$ \\
\hline 0055008 & a.. Cardiac muscle tissue morphogenesis & $1.89 \times 10^{-4}$ \\
\hline 0055010 & 3... Ventricular cardiac muscle morphogenesis & $8.12 \times 10^{-5}$ \\
\hline 0007517 & :................ Muscle organ development & $5 \cdot 75 \times 10^{-4}$ \\
\hline 0007522 & ... Visceral muscle development & $9 \cdot 11 \times 10^{-3}$ \\
\hline 0060415 & ......... Muscle tissue morphogenesis & $1 \cdot 89 \times 10^{-4}$ \\
\hline 0003007 & :............................ Heart morphogenesis & $3 \cdot 16 \times 10^{-3}$ \\
\hline 0048747 & 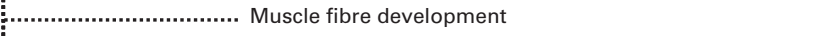 & $9 \cdot 81 \times 10^{-3}$ \\
\hline 0043462 & (ative & $1.30 \times 10^{-3}$ \\
\hline
\end{tabular}

(b)
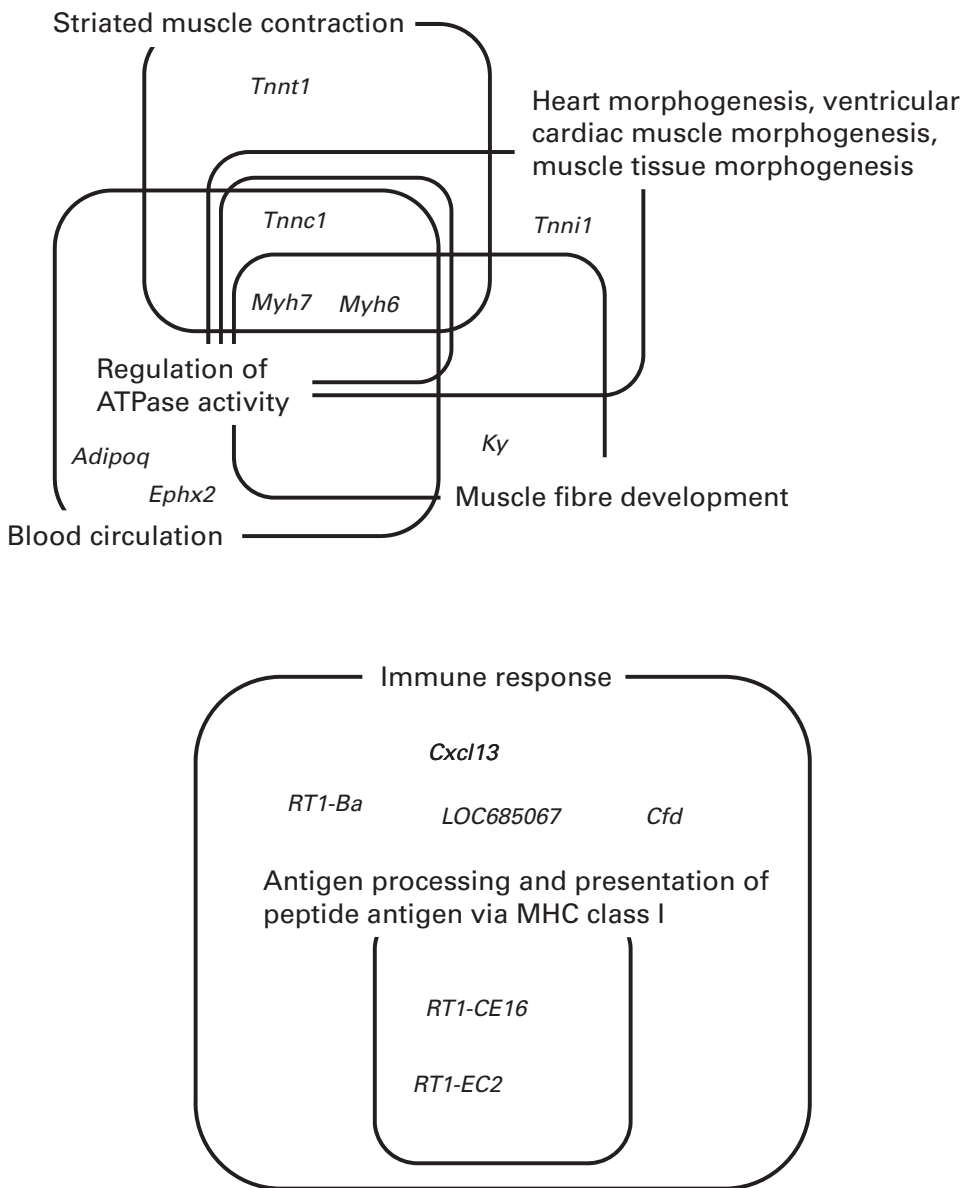

Fig. 2. Significantly enriched Gene Ontology $(\mathrm{GO})$ terms $(P<0.01)$ found in the top seventy down-regulated genes in the whey protein hydrolysate (WPH) group (a). Venn and Euler diagrams represent the association of down-regulated genes with multiple GO terms caused by WPH (b). For full names of gene symbols, see online supplementary Table S2. MHC, major histocompatibility complex. 
Fig. 3. Analysis of an Ingenuity Pathways Analysis-generated gene network. The top functions of this network are cell cycle, cellular development and lipid metabolism. The intensity of the node colour indicates the degree of up-regulation (red) or down-regulation (green). Nodes are displayed using various shapes $(\diamond$, enzyme; $\nabla$, kinase; $\square$, ligand-dependent nuclear receptor; $\odot$, transcription regulator; $\square$, transporter; $\odot$, complex/group; $\circ$, other) that represent the functional class of the gene product. Relationships: _ , direct interaction; -...., indirect interaction. For full names of gene symbols, see online supplementary Tables S1 and S2.

out using Student's $t$ test. Differences between the groups were considered to be statistically significant at $P<0.05$.

\section{Results}

\section{DNA microarray analysis of gene expression}

DFW-quantified microarray data were subjected to principal component analysis. Each experimental group formed a distinct cluster (see online supplementary Fig. S1), which indicated that WPH induced changes in the gene expression profile in the rat epitrochlearis muscle compared with the AAM.

To identify DEG in response to the ingestion of WPH, the rank products method was applied to the DFW-quantified data. The rank products method combined with a DFW preprocessing algorithm has been shown to be one of the best ways to accurately detect $\mathrm{DEG}^{(32)}$. Applying a significance value of $<0.05$ for the false discovery rate, we identified 161 up-regulated probe sets (134 genes) and seventy downregulated probe sets (fifty-five genes) in the WPH group compared with the AAM group. The full list of DEG is shown in online supplementary Tables S2 and S3.

\section{Gene Ontology analysis}

DEG were classified into functional categories according to GO. GO terms that were significantly enriched within the two sets of DEG (up- and down-regulated genes) are summarised in Figs. 1(a) and 2(a), respectively. Because of the hierarchical structure of GO, the more specific categories appear deeper in the hierarchy. Therefore, categories at the lower end of the hierarchy represent the most important characteristics of DEG.

As shown in Fig. 1(a), the most specific (i.e. appearing in the deepest hierarchy) over-represented categories in the upregulated genes were 'immune response', 'negative regulation of cytokine production', 'positive regulation of transcription from RNA polymerase II promoter', 'negative regulation of neuron differentiation', 'response to organic substance', 'response to mechanical stimulus', 'response to wounding' and 'striated muscle tissue development'. A single gene is 
Fig. 4. Analysis of an Ingenuity Pathways Analysis-generated gene network. The top functions of this network are organ morphology, skeletal and muscular system development and function, and CVD. The intensity of the node colour indicates the degree of up-regulation (red) or down-regulation (green). Nodes are displayed using various shapes that represent the functional class of the gene product. For a description of network shapes, see the legend of Fig. 3. For full names of gene symbols, see online supplementary Tables S1 and S2.

usually annotated with multiple GO terms. Therefore, these GO terms could include common DEG(s) each other. Using DAVID, we identified forty-five genes within these eight GO terms. To show the complex interdependencies of these GO terms sharing DEG, these genes and GO terms are summarised by Venn diagrams (Fig. 1(b)). It was shown that many GO terms included and shared genes involved in muscle repair. 'Immune response', 'negative regulation of cytokine production', 'negative regulation of neuron differentiation', 'response to organic substance' and 'response to wounding' included cluster of differentiation $24(C d 24)^{(33)}$. 'Immune response' and 'response to wounding' included chemokine ( $\mathrm{C}-\mathrm{C}$ motif) ligand $7(\mathrm{Ccl} 7)$ and chemokine $(\mathrm{C}-\mathrm{X}-\mathrm{C}$ motif) ligand $1(\mathrm{CxCl} 1)^{(34,35)}$. 'Response to organic substance', 'response to mechanical stimulus' and 'response to wounding' included chemokine (C-C motif) ligand $2(\mathrm{Ccl} 2)^{(34)}$.

'Positive regulation of transcription from RNA polymerase II promoter' included some key elements in skeletal muscle development such as nuclear receptor subfamily 4, group A, member 3 (Nr4a3), early growth response 1 (Egr1), Jun B proto-oncogene (Junb), nuclear receptor subfamily 4, group A, member 1 (Nr4a1), Cbp/p300-interacting transactivator, with
Glu/Asp-rich carboxy-terminal domain, 2 (Cited2), and SIX homeobox 1 (Six1). We have shown that WPH activates mTOR signalling ${ }^{(15)}$. In addition to translation initiation, mTOR regulates the function of transcription factors and gene expression ${ }^{(36)}$. Therefore, these results are consistent with those of our previous study. Thus, we focused on these transcription-related genes for subsequent investigation.

In the down-regulated gene category, the most specific overrepresented categories included 'immune response', 'antigen processing and presentation of peptide antigen', 'striated muscle contraction', 'blood circulation', 'ventricular cardiac muscle morphogenesis', 'muscle tissue morphogenesis', 'heart morphogenesis', 'muscle fibre development' and 'regulation of ATPase activity'. We identified fourteen genes in nine GO terms enriched in the gene set down-regulated by WPH. These genes and GO terms are summarised by Venn diagrams (Fig. 2(b)).

Fig. 2(b) shows that these GO terms fell into two clusters: 'regulation of ATPase activity' and 'immune response'. In particular, myosin, heavy chain 6 (Mybo) and myosin, heavy chain $7(M y b 7)$ are core elements that commonly appeared in the categories 'striated muscle contraction', 'blood circulation', 'ventricular cardiac muscle morphogenesis', 
Table 2. Extracellular signal-regulated kinase 1/2 (ERK1/2) and hypoxia-inducible factor-1 $\alpha$ (HIF-1 $\alpha)$ target genes that showed altered expression due to the administration of whey protein hydrolysate compared with amino acids

\begin{tabular}{llllr}
\hline Upstream regulator & Gene name & Gene symbol & Up/down & FDR \\
\hline ERK1/2 & & & & \\
& Nuclear receptor subfamily 4, group A, member 3 & Nr4a3 & Up & 0 \\
& Early growth response 1 & Egr1 & Up & 0 \\
& Ankyrin repeat domain 2 (stretch responsive muscle) & Ankrd2 & Up & 0.000824 \\
& Chemokine (C-C motif) ligand 7 & Ccl7 & Up & 0.002133 \\
& Jun B proto-oncogene & Junb & Up & 0.014278 \\
& Myelocytomatosis oncogene & Myc & Up & 0.027593 \\
& Serpin peptidase inhibitor, clade E (nexin, plasminogen & Serpine1 & Up & 0.040616 \\
& activator inhibitor type 1), member 1 & & \\
& Adiponectin, C1Q and collagen domain containing & Adipoq & Down & 0.0076 \\
& Cyclin-dependent kinase inhibitor 1A & Cdkn1a & Down & 0.02713 \\
& & & & \\
HIF-1 $\alpha$ & Haem oxygenase (decycling) 1 & Hmox1 & Up & 0.004364 \\
& Nuclear receptor subfamily 4, group A, member 1 & Nr4a1 & Up & 0.017517 \\
& Serpin peptidase inhibitor, clade E (nexin, plasminogen & Serpine1 & Up & 0.040616 \\
& activator inhibitor type 1), member 1 & & & \\
\hline
\end{tabular}

FDR, false discovery rate.

'muscle tissue morphogenesis', 'heart morphogenesis', 'muscle fibre development' and 'regulation of ATPase activity'. Additionally, troponin C type 1 (Tnnc1) and troponin I type 1 (Tnni1) commonly appeared in the categories 'ventricular cardiac muscle morphogenesis', 'muscle tissue morphogenesis' and 'heart morphogenesis'. All of these genes are involved in muscle contraction. Thus, we further investigated the network of muscle contraction-related genes.

\section{Gene networks}

DEG were mapped on gene networks comprising known molecular interaction, regulation and co-expression from the literature using IPA software. These networks in Figs. 3 and 4 visualised the relative magnitude and direction of gene expression changes specific to the present study.

Fig. 3 shows the network associated with cellular development. This network includes key transcription factors in DEG such as Nr4a3, Nr4a1 and Egr1. Similarly, Fig. 4 shows the network associated with skeletal and muscular system development. Although Myb 7, Tnnc1, Tnni1 and troponin T type 1 (Tnnt1) were down-regulated, interestingly, myosin, heavy chain 3 (Myb3), which is also involved in muscle contraction, was up-regulated.

\section{Upstream regulators and transcription factors}

To investigate the regulation of DEG, upstream regulators and transcription factors were predicted using IPA and TFactS, respectively. First, using IPA, we found that ERK1/2 regulated nine DEG (Table 2). Of the ERK1/2 target genes, Nr4a3, Egr1, ankyrin repeat domain 2 (stretch responsive muscle) (Ankrd2), Ccl7, Junb, myelocytomatosis oncogene (Myc) and serpin peptidase inhibitor, clade $\mathrm{E}$ (nexin, plasminogen activator inhibitor type 1), member 1 (Serpine1) were up-regulated. $N r 4 a 3$ and $J u n b$ are involved in the maintenance of muscle mass $^{(37,38)}$. Egr1 is a potential mediator of mitochondrial biogenesis ${ }^{(39)}$. Ankrd2 is involved in the expression of genes such as titin (Ttn), cysteine- and glycine-rich protein 3 (Csrp3), and myogenic differentiation $1(\operatorname{Myod} 1)^{(40)}$. The Myc protein network modulates the transcription of genes associated with glucose and energy metabolism ${ }^{(41)}$. Serpine 1 regulates fibrinolysis and proteolysis ${ }^{(42)}$. In contrast, adiponectin, C1Q and collagen domain containing (Adipoq) and cyclin-dependent kinase inhibitor 1A (Cdkn1a) were down-regulated. Adipoq selectively inhibits the ERK1/2 pathway ${ }^{(43)}$. Cdkn $1 a$ has been considered as an effector of anti-apoptotic processes ${ }^{(44)}$.

Second, using TFactS, we found that HIF-1 $\alpha$ regulated three DEG (Table 2). All of the HIF-1 $\alpha$ target genes (Nr4a1, Serpine1 and haem oxygenase (decycling) 1 (Hmox1)) were upregulated. Nr4a1 is thought to be involved in the utilisation of glucose and fatty acids ${ }^{(45)}$. Hmox 1 is normally expressed at very low levels, but increased greatly after exhaustive exercise ${ }^{(46)}$.

\section{Western blotting}

Finally, we analysed the phosphorylation of ERK1/2 and protein levels of HIF- $1 \alpha$ to determine whether WPH activated these proteins. ERK1/2 is activated by phosphorylation ${ }^{(47,48)}$, whereas protein levels of HIF- $1 \alpha$ are rapidly up-regulated by specific stimuli (e.g. hypoxia) and induce the expression of target genes $^{(49)}$. The WPH group had greater phosphorylation of ERK1/2 compared with the AAM group (Fig. 5(a)). The WPH group also demonstrated a significant increase in the protein levels of HIF-1 $\alpha$ compared with the AAM group (Fig. 5(b)).

\section{Discussion}

We have previously found that the WPH group showed significantly higher muscle protein synthesis after exercise compared with the AAM group ${ }^{(15)}$. To investigate the novel effects of WPH on global gene expression, DNA microarray analysis was employed, with the AAM as the control. Principal component analysis of the DNA microarray data revealed that there were marked differences in the gene expression profiles of skeletal muscle between the two groups.

We found eight specific GO terms that were significantly enriched in the set of up-regulated genes (Fig. 1(b)). 
(a)
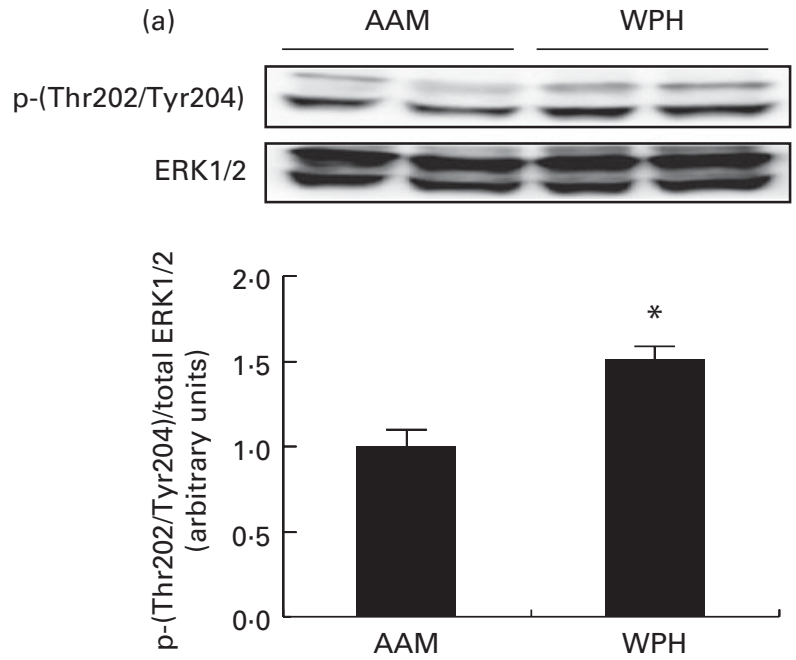

(b)
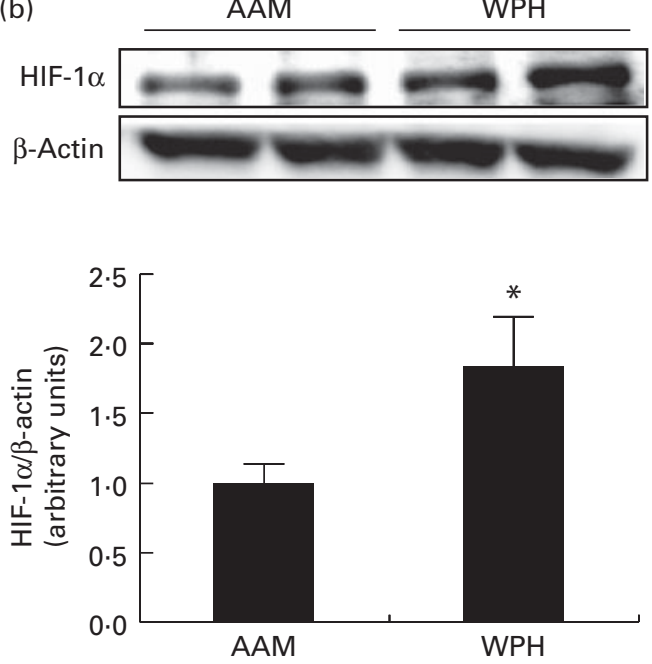

Fig. 5. Phosphorylated extracellular signal-regulated kinase $1 / 2$ (ERK1/2) (a) and hypoxia-inducible factor-1 $\alpha$ (HIF-1 $\alpha$ ) (b) levels in the skeletal muscle of rats administered a diet containing whey protein hydrolysate (WPH) or an amino acid mixture (AAM), measured $1 \mathrm{~h}$ after swimming exercise. Phosphorylated ERK1/2 was normalised to total ERK1/2, and HIF- $1 \alpha$ was normalised to $\beta$-actin. Values are means $(n 8)$, with their standard errors represented by vertical bars. ${ }^{*}$ Mean value was significantly different from that of the AAM group $(P<0.05$; Student's $t$ test).

These GO terms included some key elements such as $C d 24$, Ccl2, $\mathrm{Ccl} 7$ and $\mathrm{Cxcl} 1$ involved in post-exercise muscle repair $^{(33-35)}$. These results indicate that WPH induces more rapid muscle recovery after exercise than does the AAM. Furthermore, we found that among the GO terms appearing in the deepest hierarchy, 'positive regulation of transcription from RNA polymerase II promoter' included important transcription factors in skeletal muscle. Therefore, we focused on this GO term. To clarify the interaction of genes included in this GO term, we investigated the gene network. The network associated with cellular development showed that some transcription factors (Nr4a3, Nr4a1 and Egr1) interacted with Akt, which is a key enzyme in glucose metabolism and muscle protein synthesis ${ }^{(7,48)}$. We have previously shown that ingestion of WPH induces a greater increase in muscle glycogen levels via activation of Akt compared with ingestion of glucose alone after exercise ${ }^{(14)}$. This result indicates that activation of Akt induced by WPH is involved in the upregulation of the genes Nr4a3, Nr4a1 and Egr1. These genes are metabolism-related genes ${ }^{(39,45)}$. In the present study, the gene network analysis revealed that these genes interacted with some glucoregulatory enzymes, such as hexokinase $^{(50)}$, pyruvate kinase ${ }^{(51)}$ and phosphofructokinase ${ }^{(52)}$. Thus, these up-regulated genes were possibly involved in the increase in muscle glycogen content caused by WPH.

We found nine GO terms enriched in the gene set downregulated by WPH, and these GO terms fell into two clusters. Of these two clusters, one included some genes encoding skeletal muscle fibres, such as Myb7, Tnni1, Tnnc1 and Tnnt1. Investigating the gene network of these muscle fibrerelated genes, we found that $M y b 3$ encoding skeletal muscle fibre protein was up-regulated (Fig. 4). Rodent skeletal muscle is composed of four distinct fibre types. Type I, IIa, IIx and IIb fibres differ with respect to contractile function, the number of mitochondria and metabolism ${ }^{(53,54)}$. The slow type I fibres can sustain prolonged low power work, contain more mitochondria and exhibit higher rates of fat oxidative metabolism. The fast type IIx and IIb fibres are adapted to brief and intense contractions and contain fewer mitochondria and generate energy mainly through glycolysis. The type IIa fibres are oxidoglycolytic and exhibit an intermediate contractile function. Interestingly, all of the down-regulated genes (Myb 7, Tnni1, Tnnc1 and Tnnt1) encoded type I fibres ${ }^{(53)}$. In contrast, $M y h 3$ was expressed during the embryonic period ${ }^{(55)}$. We also found that WPH up-regulated a key transcription factor, Six1. Six1 can reprogram adult muscle from the slow-twitch phenotype into the fast-twitch phenotype ${ }^{(56)}$. In the present study, we used the epitrochlearis muscle for DNA microarray analysis and the triceps muscle for analysing the FSR and protein levels of ERK1/2 and HIF-1 $\alpha$. Both the epitrochlearis and triceps were recruited during swimming exercise in rats ${ }^{(57)}$ In addition, both the epitrochlearis and triceps are highly glycolytic muscles with approximately $90 \%$ type II fibres ${ }^{(58,59)}$. Thus, both have similar functions and fibre compositions. Taken together, it is concluded that WPH can enhance adaptation to exercise by increasing type II fibres in the epitrochlearis and triceps to a greater extent than can the AAM.

The present microarray data also showed that two key factors, ERK1/2 and HIF-1 $\alpha$, regulated nine genes and three genes in DEG, respectively. In addition, we showed that WPH caused greater phosphorylation of ERK1/2 and increased protein levels of HIF- $1 \alpha$ compared with the AAM. These results indicate that ingestion of WPH might regulate gene expression via activation of ERK1/2 and HIF-1 $\alpha$. The possible pathway of the regulation of these genes by ERK1/2 and/or HIF- $1 \alpha$ is shown in Fig. 6. The ERK1/2 signalling pathway is important, as it is involved in the regulation of translation initiation and elongation following muscle contraction ${ }^{(47)}$. Although the cross-talk of the ERK1/2 and mTOR signalling pathways has been identified, it is believed that the effect of nutrients on muscle protein turnover is mainly due to the activation of the mTOR signalling pathway $^{(48)}$. In our previous study, we have shown that WPH strongly stimulated mTOR signalling compared with the AAM. 


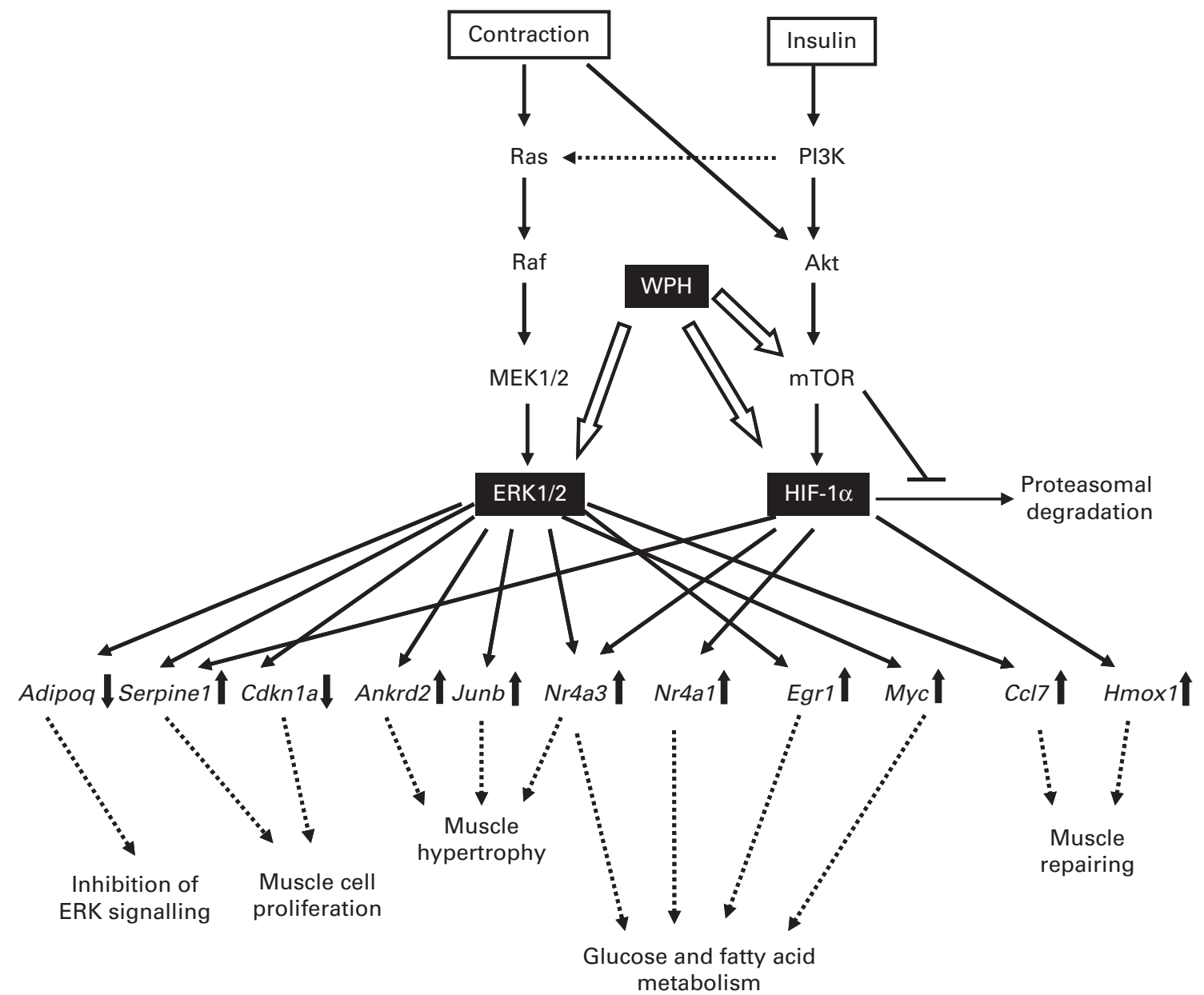

Fig. 6. Possible pathway of the regulation of gene expression by whey protein hydrolysate (WPH) via activation of extracellular signal-regulated kinase $1 / 2$ (ERK1/2) and hypoxia-inducible factor-1 $\alpha$ (HIF-1 $\alpha$ ). MEK1/2, mitogen-activated protein kinase 1/2; PI3K, phosphatidylinositol 3-kinase; mTOR, mammalian target of rapamycin. For full names of gene symbols, see Table 2.

According to Karlsson et al. ${ }^{(60)}$, BCAA activate translation initiation that is dependent on mTOR signalling, but not on ERK1/2 signalling in humans. However, the present results indicated that WPH activated ERK1/2 signalling as well as mTOR signalling. Recently, Motobayashi et al. ${ }^{(43)}$ showed that adiponectin inhibits insulin-like growth factor-1-induced cell migration by the suppression of ERK1/2 activation. Therefore, the results of the present study suggest that WPH keeps the activated state of ERK1/2 signalling by the down-regulation of Adipoq expression. In contrast, HIF- $1 \alpha$ mediates the transcriptional response to hypoxia ${ }^{(61)}$. It is notable that HIF- $1 \alpha$ expression and function are regulated by mTOR signalling ${ }^{(61)}$. Although it remains unclear whether specific nutrients activate HIF- $1 \alpha$, it is possible that WPH activates HIF- $1 \alpha$ via activation of mTOR signalling. The findings of the present study provide new insights into the effects of nutrients on mTOR and ERK1/2 signalling.

The three up-regulated genes Nr4a3 ${ }^{(37)}, J u n b^{(38)}$ and Ankrd2 ${ }^{(62,63)}$ related to muscle hypertrophy are regulated by HIF-1 $\alpha$ and/or ERK1/2. Therefore, we hypothesise that there is a relationship between the series of gene expression changes and the greater increase in muscle protein synthesis caused by the ingestion of WPH. Moreover, two genes related to muscle repair, $\mathrm{Ccl} 7$ and Hmox1, are also regulated by ERK1/2 or
HIF-1 $\alpha$. Injured muscle tissue requires $C c l 2$ expression for repair. It is possible that $\mathrm{Ccl} 7$ also plays an important role in the response to acute skeletal muscle injury, especially in the absence of $\mathrm{Ccl} 2^{(34)}$. Although the physiological role of Hmox1 in muscle has not been established, Pilegaard et al. ${ }^{(46)}$ suggested that Hmox1 is induced in response to exercise to help minimise intracellular damage by free radicals, similar to the protective role of Hmox1 in other tissues. These results indicated that WPH induces more rapid muscle recovery via activation of ERK1/2 and HIF-1 $\alpha$.

The amino acid compositions of the two solutions (WPH and $\mathrm{AAM}$ ) used in the present study were equivalent and plasma amino acid levels did not differ between the two groups, although the effects of the two diets on gene expression varied. Therefore, it is possible that active components, e.g. bioactive peptide, contained in WPH had an effect on gene expression. Previously, we noted that plasma levels of BCAA-containing bioactive peptides were elevated markedly after ingestion of $\mathrm{WPH}^{(15,19)}$. Additional studies are therefore required to determine whether these dipeptides themselves caused the effect of WPH on gene expression.

In conclusion, in the present study of post-exercise rat skeletal muscle, we identified 189 genes whose expression 
was altered by the ingestion of WPH compared with that observed following ingestion of the AAM. Furthermore, we found two key upstream proteins, ERK1/2 and HIF- $1 \alpha$, that are predicted to regulate gene expression after ingestion of WPH. To our knowledge, the present study is the first to show that, compared with an AAM, ingestion of WPH induces changes in the gene expression profile via activation of ERK1/2 and HIF- $1 \alpha$. The present study only covered a $1 \mathrm{~h}$ range after ingestion; changes in the gene expression profile need to be studied over time to uncover the mechanisms underlying the superior repair effects caused by WPH compared with the AAM.

\section{Supplementary material}

To view supplementary material for this article, please visit http://dx.doi.org/10.1017/S0007114514000233

\section{Acknowledgements}

The present study received no funding.

The authors' contributions are as follows: A. K. designed the research; A. K., T. F., Y. N., K. A., K. K. and S. I. conducted the research; A. K., T. I., F. S., K. N., Y. N. and K. A. analysed the data; A. K. wrote the paper, and had primary responsibility for the final content. All authors read and approved the final manuscript.

The authors have no conflicts of interest to declare.

\section{References}

1. Philp A, Hargreaves M \& Baar K (2012) More than a store: regulatory roles for glycogen in skeletal muscle adaptation to exercise. Am J Physiol Endocrinol Metab 302, E1343-E1351.

2. Walker DK, Dickinson JM, Timmerman KL, et al. (2011) Exercise, amino acids, and aging in the control of human muscle protein synthesis. Med Sci Sports Exerc 43, 2249-2258.

3. Dreyer HC, Fujita S, Cadenas JG, et al. (2006) Resistance exercise increases AMPK activity and reduces 4E-BP1 phosphorylation and protein synthesis in human skeletal muscle. J Physiol 576, 613-624.

4. Zawadzki KM, Yaspelkis BB 3rd \& Ivy JL (1992) Carbohydrate-protein complex increases the rate of muscle glycogen storage after exercise. J Appl Physiol 72, 1854-1859.

5. Ivy JL, Goforth HW Jr, Damon BM, et al. (2002) Early postexercise muscle glycogen recovery is enhanced with a carbohydrateprotein supplement. J Appl Physiol 93, 1337-1344.

6. Armstrong JL, Bonavaud SM, Toole BJ, et al. (2001) Regulation of glycogen synthesis by amino acids in cultured human muscle cells. J Biol Chem 276, 952-956.

7. Peyrollier K, Hajduch E, Blair AS, et al. (2000) L-Leucine availability regulates phosphatidylinositol 3-kinase, p70 S6 kinase and glycogen synthase kinase-3 activity in L6 muscle cells: evidence for the involvement of the mammalian target of rapamycin (mTOR) pathway in the L-leucine-induced up-regulation of system A amino acid transport. Biochem J 350, 361-368.

8. Doi M, Yamaoka I, Nakayama M, et al. (2005) Isoleucine, a blood glucose-lowering amino acid, increases glucose uptake in rat skeletal muscle in the absence of increases in AMP-activated protein kinase activity. J Nutr 135, 2103-2108.

9. Anthony JC, Anthony TG \& Layman DK (1999) Leucine supplementation enhances skeletal muscle recovery in rats following exercise. J Nutr 129, 1102-1106.
10. Churchward-Venne TA, Burd NA, Mitchell CJ, et al. (2012) Supplementation of a suboptimal protein dose with leucine or essential amino acids: effects on myofibrillar protein synthesis at rest and following resistance exercise in men. J Physiol 590, 2751-2765.

11. Anthony JC, Anthony TG, Kimball SR, et al. (2000) Orally administered leucine stimulates protein synthesis in skeletal muscle of postabsorptive rats in association with increased eIF4F formation. J Nutr 130, 139-145.

12. Anthony JC, Yoshizawa F, Anthony TG, et al. (2000) Leucine stimulates translation initiation in skeletal muscle of postabsorptive rats via a rapamycin-sensitive pathway. J Nutr 130, 2413-2419.

13. Norton LE, Layman DK, Bunpo P, et al. (2009) The leucine content of a complete meal directs peak activation but not duration of skeletal muscle protein synthesis and mammalian target of rapamycin signaling in rats. J Nutr 139, 1103-1109.

14. Morifuji M, Kanda A, Koga J, et al. (2010) Post-exercise carbohydrate plus whey protein hydrolysates supplementation increases skeletal muscle glycogen level in rats. Amino Acids 38, 1109-1115.

15. Kanda A, Nakayama K, Fukasawa T, et al. (2013) Post-exercise whey protein hydrolysate supplementation induces a greater increase in muscle protein synthesis than its constituent amino acid content. BrJ Nutr 110, 981-987.

16. Morifuji M, Koga J, Kawanaka K, et al. (2009) Branchedchain amino acid-containing dipeptides, identified from whey protein hydrolysates, stimulate glucose uptake rate in L6 myotubes and isolated skeletal muscles. J Nutr Sci Vitaminol (Tokyo) 55, 81-86.

17. Rowlands DS, Thomson JS, Timmons BW, et al. (2011) Transcriptome and translational signaling following endurance exercise in trained skeletal muscle: impact of dietary protein. Physiol Genomics 43, 1004-1020.

18. Sonou T, Higuchi M \& Terada S (2008) An acute bout of high-intensity intermittent swimming induces glycogen supercompensation in rat skeletal muscle. Eur J Sport Sci 8, 413-420.

19. Morifuji M, Ishizaka M, Baba S, et al. (2010) Comparison of different sources and degrees of hydrolysis of dietary protein: effect on plasma amino acids, dipeptides, and insulin responses in human subjects. J Agric Food Chem 58, 8788-8797.

20. Suyama T, Okada S, Ishijima T, et al. (2012) High phosphorus diet-induced changes in NaPi-IIb phosphate transporter expression in the rat kidney: DNA microarray analysis. PLOS ONE 7, e29483.

21. Chen Z, McGee M, Liu Q, et al. (2007) A distribution free summarization method for Affymetrix GeneChip arrays. Bioinformatics 23, 321-327.

22. $\mathrm{R}$ Development Core Team (2006) A Language and Environment for Statistical Computing. Vienna: R Foundation for Statistical Computing.

23. Gentleman RC, Carey VJ, Bates DM, et al. (2004) Bioconductor: open software development for computational biology and bioinformatics. Genome Biol 5, R80.

24. Kachigan S (1996) Statistical Analysis. New York: Radius Press.

25. Breitling R, Armengaud P, Amtmann A, et al. (2004) Rank products: a simple, yet powerful, new method to detect differentially regulated genes in replicated microarray experiments. FEBS Lett 573, 83-92.

26. Huang da W, Sherman BT \& Lempicki RA (2009) Systematic and integrative analysis of large gene lists using DAVID bioinformatics resources. Nat Protoc 4, 44-57. 
27. Binns D, Dimmer E, Huntley R, et al. (2009) QuickGO: a web-based tool for Gene Ontology searching. Bioinformatics 25, 3045-3046.

28. Hosack DA, Dennis G Jr, Sherman BT, et al. (2003) Identifying biological themes within lists of genes with EASE. Genome Biol 4, R70.

29. Essaghir A, Toffalini F, Knoops L, et al. (2010) Transcription factor regulation can be accurately predicted from the presence of target gene signatures in microarray gene expression data. Nucleic Acids Res 38, e120.

30. Notas G, Alexaki VI, Kampa M, et al. (2012) APRIL binding to BCMA activates a JNK2-FOXO3-GADD45 pathway and induces a $\mathrm{G} 2 / \mathrm{M}$ cell growth arrest in liver cells. J Immunol 189, $4748-4758$.

31. Morifuji M, Kanda A, Koga J, et al. (2011) Preexercise ingestion of carbohydrate plus whey protein hydrolysates attenuates skeletal muscle glycogen depletion during exercise in rats. Nutrition 27, 833-837.

32. Kadota K, Nakai Y \& Shimizu K (2009) Ranking differentially expressed genes from Affymetrix gene expression data: methods with reproducibility, sensitivity, and specificity. Algorithms Mol Biol 4, 7.

33. Higuchi I, Kawai H, Kawajiri M, et al. (1999) Statistically significant differences in the number of CD24 positive muscle fibers and satellite cells between sarcoglycanopathy and age-matched Becker muscular dystrophy patients. Intern Med 38, 412-415.

34. Lu H, Huang D, Ransohoff RM, et al. (2011) Acute skeletal muscle injury: CCL2 expression by both monocytes and injured muscle is required for repair. FASEB J 25, 3344-3355.

35. Roseguini BT, Arce-Esquivel AA, Newcomer SC, et al. (2011) Impact of a single session of intermittent pneumatic leg compressions on skeletal muscle and isolated artery gene expression in rats. Am J Physiol Regul Integr Comp Physiol 301, R1658-R1668.

36. Sengupta S, Peterson TR \& Sabatini DM (2010) Regulation of the mTOR complex 1 pathway by nutrients, growth factors, and stress. Mol Cell 40, 310-322.

37. Pearen MA, Ryall JG, Maxwell MA, et al. (2006) The orphan nuclear receptor, NOR-1, is a target of beta-adrenergic signaling in skeletal muscle. Endocrinology 147, 5217-5227.

38. Raffaello A, Milan G, Masiero E, et al. (2010) JunB transcription factor maintains skeletal muscle mass and promotes hypertrophy. J Cell Biol 191, 101-113.

39. Irrcher I \& Hood DA (2004) Regulation of Egr-1, SRF, and Sp1 mRNA expression in contracting skeletal muscle cells. J Appl Physiol 97, 2207-2213.

40. Barash IA, Bang ML, Mathew L, et al. (2007) Structural and regulatory roles of muscle ankyrin repeat protein family in skeletal muscle. Am J Physiol Cell Physiol 293, C218-C227.

41. Gohil K \& Brooks GA (2012) Exercise tames the wild side of the Myc network: a hypothesis. Am J Physiol Endocrinol Metab 303, E18-E30.

42. Lijnen HR (2005) Pleiotropic functions of plasminogen activator inhibitor-1. J Thromb Haemost 3, 35-45.

43. Motobayashi Y, Izawa-Ishizawa Y, Ishizawa K, et al. (2009) Adiponectin inhibits insulin-like growth factor-1-induced cell migration by the suppression of extracellular signalregulated kinase $1 / 2$ activation, but not Akt in vascular smooth muscle cells. Hypertens Res 32, 188-193.

44. Ishido M, Kami K \& Masuhara M (2004) In vivo expression patterns of MyoD, p21, and $\mathrm{Rb}$ proteins in myonuclei and satellite cells of denervated rat skeletal muscle. Am J Physiol Cell Physiol 287, C484-C493.
45. Kawasaki E, Hokari F, Sasaki M, et al. (2009) Role of local muscle contractile activity in the exercise-induced increase in NR4A receptor mRNA expression. J Appl Physiol 106, 1826-1831.

46. Pilegaard H, Ordway GA, Saltin B, et al. (2000) Transcriptional regulation of gene expression in human skeletal muscle during recovery from exercise. Am $J$ Physiol Endocrinol Metab 279, E806-E814.

47. Goodyear LJ, Chang PY, Sherwood DJ, et al. (1996) Effects of exercise and insulin on mitogen-activated protein kinase signaling pathways in rat skeletal muscle. Am J Physiol 271, E403-E408.

48. Drummond MJ, Dreyer HC, Fry CS, et al. (2009) Nutritional and contractile regulation of human skeletal muscle protein synthesis and mTORC1 signaling. J Appl Physiol 106, 1374-1384.

49. Martorell L, Gentile M, Rius J, et al. (2009) The hypoxia-inducible factor 1/NOR-1 axis regulates the survival response of endothelial cells to hypoxia. Mol Cell Biol 29, 5828-5842.

50. Shulman RG, Bloch G \& Rothman DL (1995) In vivo regulation of muscle glycogen synthase and the control of glycogen synthesis. Proc Natl Acad Sci U S A 92, 8535-8542.

51. Yamada K \& Noguchi T (1999) Nutrient and hormonal regulation of pyruvate kinase gene expression. Biochem J337, 1-11.

52. Mayes PA (1993) Intermediary metabolism of fructose. Am J Clin Nutr 58, 754S-765S

53. de Wilde J, Hulshof MF, Boekschoten MV, et al. (2010) The embryonic genes Dkk3, Hoxd8, Hoxd9 and Tbx1 identify muscle types in a diet-independent and fiber-type unrelated way. BMC Genomics 11, 176.

54. Booth FW \& Thomason DB (1991) Molecular and cellular adaptation of muscle in response to exercise: perspectives of various models. Physiol Rev 71, 541-585.

55. Eller M, Stedman HH, Sylvester JE, et al. (1989) Human embryonic myosin heavy chain cDNA. Interspecies sequence conservation of the myosin rod, chromosomal locus and isoform specific transcription of the gene. FEBS Lett 256, 21-28.

56. Grifone R, Laclef C, Spitz F, et al. (2004) Six1 and Eya1 expression can reprogram adult muscle from the slowtwitch phenotype into the fast-twitch phenotype. Mol Cell Biol 24, 6253-6267.

57. Terada S \& Tabata I (2004) Effects of acute bouts of running and swimming exercise on PGC-1alpha protein expression in rat epitrochlearis and soleus muscle. Am J Physiol Endocrinol Metab 286, E208-E216.

58. Rodnick KJ, Henriksen EJ, James DE, et al. (1992) Exercise training, glucose transporters, and glucose transport in rat skeletal muscles. Am J Physiol 262, C9-C14.

59. Fuentes I, Cobos AR \& Segade LA (1998) Muscle fibre types and their distribution in the biceps and triceps brachii of the rat and rabbit. J Anat 192, 203-210.

60. Karlsson HK, Nilsson PA, Nilsson J, et al. (2004) Branchedchain amino acids increase p70S6k phosphorylation in human skeletal muscle after resistance exercise. Am J Physiol Endocrinol Metab 287, E1-E7.

61. Semenza GL (2009) Regulation of oxygen homeostasis by hypoxia-inducible factor 1. Physiology (Bethesda) 24, 97-106.

62. Kemp TJ, Sadusky TJ, Saltisi F, et al. (2000) Identification of Ankrd2, a novel skeletal muscle gene coding for a stretchresponsive ankyrin-repeat protein. Genomics $\mathbf{6 6}, 229-241$.

63. Tsukamoto Y, Hijiya N, Yano S, et al. (2008) Arpp/Ankrd2, a member of the muscle ankyrin repeat proteins (MARPs), translocates from the I-band to the nucleus after muscle injury. Histochem Cell Biol 129, 55-64. 